International Journal of Pure and Applied Mathematics

Volume 113 No. 1 2017, 93-102

ISSN: 1311-8080 (printed version); ISSN: 1314-3395 (on-line version)

url: http://www.ijpam.eu

doi: 10.12732/ijpam.v113i1.9

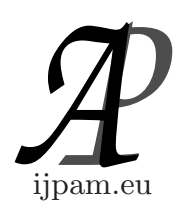

\title{
ON SEMI GENERALIZED STAR $b$-CLOSED \\ MAP IN TOPOLOGICAL SPACES
}

\author{
S. Sekar ${ }^{1 \S}$, B. Jothilakshmi ${ }^{2}$ \\ ${ }^{1}$ Department of Mathematics \\ Government Arts College (Autonomous) \\ Salem, 636 007, Tamil Nadu, INDIA \\ ${ }^{2}$ Department of Mathematics \\ Government Arts College (Autonomous) \\ Coimbatore, 641 045, Tamil Nadu, INDIA
}

\begin{abstract}
In this paper, we introduce a new class of semi generalized star $b$-closed map and study some of their properties as well as inter relationship with other closed maps.
\end{abstract}

AMS Subject Classification: $54 \mathrm{C} 05,54 \mathrm{C} 08,54 \mathrm{C} 10$

Key Words: $s g^{*} b$-closed map, $b$-closed map, $g b$ closed map and $r g b$ closed map

\section{Introduction}

Different types of closed and open mappings were studied by various researchers. In 1996, Andrijevic introduced new type of set called $b$ - open set. A.A.Omari and M.S.M. Noorani introduced and studied $b$ - closed map.

The aim of this paper is to introduce semi generalized star $b$ - closed map and to continue the study of its relationship with various generalized closed maps. Through out this paper $(X, \tau)$ and $(Y, \sigma)$ represent the non-empty topological spaces on which no separation axioms are assumed, unless otherwise mentioned.

Received: November 5, 2016

Revised: January 24, 2017

Published: February 28, 2017

(c) 2017 Academic Publications, Ltd. url: www.acadpubl.eu

$\S_{\text {Correspondence author }}$ 
Let $A \subseteq X$, the closure of $A$ and interior of $A$ will be denoted by $\operatorname{cl}(A)$ and $\operatorname{int}(A)$ respectively, union of all $b$ - open sets $X$ contained in $A$ is called $b$ interior of $A$ and it is denoted by $\operatorname{bint}(A)$, the intersection of all $b$ - closed sets of $X$ containing $A$ is called $b$ - closure of $A$ and it is denoted by $b c l(A)$.

\section{Preliminaries}

Definition 2.1. Let A subset $A$ of a topological space $(X, \tau)$, is called $[1)]$

1. a pre-open set [15] if $A \subseteq \operatorname{int}(\operatorname{cl}(A))$.

2. a semi-open set [12] if $A \subseteq \operatorname{cl}(\operatorname{int}(A))$.

3. a $\alpha$-open set [16] if $A \subseteq \operatorname{int}(\operatorname{cl}(\operatorname{int}(A)))$.

4. a $\alpha$ generalized closed set (briefly $\alpha g$ - closed) [13] if $\alpha c l(A) \subseteq U$ whenever $A \subseteq U$ and $U$ is open in $X$.

5. a generalized $*$ closed set (briefly $g^{*}$-closed)[19] if $\operatorname{cl}(A) \subseteq U$ whenever $A \subseteq U$ and $U$ is $\hat{g}$ open in $X$.

6. a generalized $b$ - closed set (briefly $g b$ - closed) [1] if $b c l(A) \subseteq U$ whenever $A \subseteq U$ and $U$ is open in $X$.

7. a generalized semi-pre closed set (briefly $g s p$ - closed) [8] if $\operatorname{spcl}(A) \subseteq U$ whenever $A \subseteq U$ and $U$ is open in $X$.

8. a generalized pre- closed set (briefly gp- closed) [9] if $p c l(A) \subseteq U$ whenever $A \subseteq U$ and $U$ is open in $X$.

9. a generalized semi- closed set (briefly $g s$ - closed) [8] if $\operatorname{scl}(A) \subseteq U$ whenever $A \subseteq U$ and $U$ is open in $X$.

10. a semi generalized closed set (briefly $s g$ - closed) [5] if $\operatorname{scl}(A) \subseteq U$ whenever $A \subseteq U$ and $U$ is semi open in $X$.

11. a generalized pre regular closed set (briefly gpr-closed) [9] if $\operatorname{pcl}(A) \subseteq U$ whenever $A \subseteq U$ and $U$ is regular open in $X$.

12. a semi generalized $b$ - closed set (briefly $s g b$ - closed) [10] if $b c l(A) \subseteq U$ whenever $A \subseteq U$ and $U$ is semi open in $X$. 
13. a $\ddot{g}$ - closed set [18] if $c l(A) \subseteq U$ whenever $A \subseteq U$ and $U$ is $s g$ open in $X$.

14. a semi generalized star $b$ - closed set (briefly $s g^{*} b$ - closed) [19] if $b c l(A) \subseteq U$ whenever $A \subseteq U$ and $U$ is semi open in $X$.

\section{On Semi Generalized $s g^{*} b$-Closed Map}

In this section, we introduce semi generalized star $b$ - closed map $\left(s g^{*} b\right.$ - closed $)$ in topological spaces by using the notions of $s g^{*} b$ - closed sets and study some of their properties.

Definition 3.1. Let $X$ and $Y$ be two topological spaces. A map $f$ : $(X, \tau) \rightarrow(Y, \delta)$ is called semi generalized star $b$ - closed (briefly, $s g^{*} b$ - closed map) if the image of every closed set in $X$ is $s g^{*} b$-closed in $Y$.

Theorem 3.2. Every closed map is $s g^{*} b$ - closed but not conversely.

Proof. Let $f:(X, \tau) \rightarrow(Y, \delta)$ is closed map and $V$ be an closed set in $X$ then $f(V)$ is closed in $Y$. Hence $s g^{*} b$ - closed in $Y$. Then $f$ is $s g^{*} b$ - closed.

The converse of above theorem need not be true as seen from the following example.

Example 3.3. Consider $X=Y=\{a, b, c\}, \tau=\{X, \phi,\{a\},\{b\},\{a, b\}\}$ and $\sigma=\{Y, \phi,\{a, b\}\}$. Let $f:(X, \tau) \rightarrow(Y, \sigma)$ be defined by $f(a)=b, f(b)=$ $c, f(c)=a$. The map is $s g^{*} b$ - closed but not closed as the image of $\{b, c\}$ in $X$ is $\{a, c\}$ is not closed in $Y$.

Theorem 3.4. Every $\ddot{g}$-closed map is $s g^{*} b$ - closed set but not conversely.

Proof. Let $f:(X, \tau) \rightarrow(Y, \sigma)$ is $\ddot{g}$ - closed map and $V$ be an closed set in $X$ then $f(V)$ is closed in $Y$. Hence $s g^{*} b$ - closed in $Y$. Then $f$ is $s g^{*} b$ - closed.

The converse of above theorem need not be true as seen from the following example.

Example 3.5. Consider $X=Y=\{a, b, c\}, \tau=\{X, \phi,\{a\},\{c\},\{a, c\}\}$ and $\sigma=\{Y, \phi,\{a, b\}\}$. Let $f:(X, \tau) \rightarrow(Y, \sigma)$ be defined by $f(a)=c, f(b)=$ $b, f(c)=a$. The map is $s g^{*} b$ - closed but not $\ddot{g}$ - closed as the image of $\{a, b\}$ in $X$ is $\{b, c\}$ is not $\ddot{g}$ - closed in $Y$.

Theorem 3.6. Every semi - closed map is $s g^{*} b$ - closed but not conversely. 
Proof. Let $f:(X, \tau) \rightarrow(Y, \sigma)$ be semi-closed map and $V$ be an closed set in $X$ then $f(V)$ is closed in $Y$. Hence $s g^{*} b$ - closed in $Y$. Then $f$ is $s g^{*} b-$ closed.

The converse of above theorem need not be true as seen from the following example.

Example 3.7. Consider $X=Y=\{a, b, c\}, \tau=\{X, \phi,\{a\},\{a, c\},\{a, b\}\}$ and $\sigma=\{Y, \phi,\{a, b\}\}$. Let $f:(X, \tau) \rightarrow(Y, \sigma)$ be defined by $f(a)=a, f(b)=$ $c, f(c)=b$. The map is $s g^{*} b$ - closed but not semi - closed as the image of $\{b, c\}$ in $X$ is $\{b, c\}$ is not semi-closed in $Y$.

Theorem 3.8. Every $\alpha$-closed map is $s g^{*} b$ - closed but not conversely.

Proof. Let $f:(X, \tau) \rightarrow(Y, \delta)$ be semi - closed map and $V$ be an closed set in $X$ then $f(V)$ is closed in $Y$. Hence $s g^{*} b$ - closed in $Y$. Then $f$ is $s g^{*} b$ closed.

The converse of above theorem need not be true as seen from the following example.

Example 3.9. Consider $X=Y=\{a, b, c\}, \tau=\{X, \phi,\{c\}\}$ and $\sigma=$ $\{Y, \phi,\{b\},\{a, b\}\}$. Let $f:(X, \tau) \rightarrow(Y, \sigma)$ be defined by $f(a)=a, f(b)=$ $b, f(c)=c$. The map is $s g^{*} b$ - closed but not $\alpha$ - closed as the image of $\{a, b\}$ in $X$ is $\{a, b\}$ is not $\alpha$ - closed in $Y$.

Theorem 3.10. Every pre-closed map is $s g^{*} b$ - closed but not conversely.

Proof. Let $f:(X, \tau) \rightarrow(Y, \delta)$ pre - closed map and $V$ be an closed set in $X$ then $f(V)$ is closed in $Y$. Hence $s g^{*} b$ - closed in $Y$. Then $f$ is $s g^{*} b$ - closed.

The converse of above theorem need not be true as seen from the following example.

Example 3.11. Consider $X=Y=\{a, b, c\}, \tau=\{X, \phi,\{c\},\{b, c\}\}$ and $\sigma=\{Y, \phi,\{b\},\{a, b\}\}$. Let $f:(X, \tau) \rightarrow(Y, \sigma)$ be defined by $f(a)=b, f(b)=$ $a, f(c)=c$. The map is $s g^{*} b$-closed but not pre-closed as the image of $\{a, b\}$ in $X$ is $\{a, b\}$ is not pre-closed in $Y$.

Theorem 3.12. Every $\alpha g$-closed map is $s g^{*} b$-closed but not conversely.

Proof. Let $f:(X, \tau) \rightarrow(Y, \sigma)$ be $\alpha g$ - closed map and $V$ be an closed set in $X$ then $f(V)$ is closed in $Y$. Hence $s g^{*} b$ - closed in $Y$. Then $f$ is $s g^{*} b-$ closed. 
The converse of above theorem need not be true as seen from the following example.

Example 3.13. Consider $X=Y=\{a, b, c\}, \tau=\{X, \phi,\{b\},\{a, b\}\}$ and $\sigma=\{Y, \phi,\{a, b\}\}$. Let $f:(X, \tau) \rightarrow(Y, \sigma)$ be defined by $f(a)=c, f(b)=$ $a, f(c)=b$. The map is $s g^{*} b$ - closed but not $\alpha g$-closed as the image of $\{c\}$ in $X$ is $\{b\}$ is not $\alpha g$ - closed in $Y$.

Theorem 3.14. Every $s g^{*} b$ - closed map is gsp-closed but not conversely.

Proof. Let $f:(X, \tau) \rightarrow(Y, \delta)$ be $s g^{*} b$ closed map and $V$ be an closed set in $X$ then $f(V)$ is closed in $Y$. Hence $g s p$ - closed in $Y$. Then $f$ is $g s p$ - closed.

The converse of above theorem need not be true as seen from the following example.

Example 3.15. Consider $X=Y=\{a, b, c\}, \tau=\{X, \phi,\{b\}\}$ and $\sigma=$ $\{Y, \phi,\{a\},\{c\},\{a, c\}\}$. Let $f:(X, \tau) \rightarrow(Y, \sigma)$ be defined by $f(a)=b, f(b)=$ $c, f(c)=a$. The map is $g s p$ - closed but not $s g^{*} b$ - closed as the image of $\{a, c\}$ in $X$ is $\{a, b\}$ is not $s g^{*} b$ - closed in $Y$.

Theorem 3.16. Every $s g^{*} b$ - closed map is $g b$-closed but not conversely.

Proof. Let $f:(X, \tau) \rightarrow(Y, \delta)$ be $s g^{*} b$ - closed map and $V$ be an closed set in $X$ then $f(V)$ is closed in $Y$. Hence $g b$ - closed in $Y$. Then $f$ is $g b$ - closed.

The converse of above theorem need not be true as seen from the following example.

Example 3.17. Consider $X=Y=\{a, b, c\}, \tau=\{X, \phi,\{a\}\}$ and $\sigma=$ $\{Y, \phi,\{a\},\{a, b\}\}$. Let $f:(X, \tau) \rightarrow(Y, \sigma)$ be defined by $f(a)=b, f(b)=$ $a, f(c)=c$. The map is $s g^{*} b$ - closed but not $g b$ - closed as the image of $\{b, c\}$ in $X$ is $\{a, c\}$ is not closed in $Y$.

Theorem 3.18. Every $s g$ - closed map is $s g^{*} b$ - closed but not conversely.

Proof. Let $f:(X, \tau) \rightarrow(Y, \delta)$ be $s g$ - closed map and $V$ be an closed set in $X$ then $f(V)$ is closed in $Y$. Hence $s g^{*} b$ - closed in $Y$. Then $f$ is $s g^{*} b-$ closed.

The converse of above theorem need not be true as seen from the following example. 
Example 3.19. Consider $X=Y=\{a, b, c\}, \tau=\{X, \phi,\{b\},\{a, c\}\}$ and $\sigma=\{Y, \phi,\{b\},\{a, b\}\}$. Let $f:(X, \tau) \rightarrow(Y, \sigma)$ be defined by $f(a)=c, f(b)=$ $b, f(c)=a$. The map is $s g$-closed but not $s g^{*} b$ - closed as the image of $\{a, c\}$ in $X$ is $\{a, b\}$ is not closed in $Y$.

Theorem 3.20. A map $f:(X, \tau) \rightarrow(Y, \sigma)$ is continuous and $s g^{*} b$ - closed set $A$ is $s g^{*} b$-closed set of $X$ then $f(A)$ is $s g^{*} b$ closed in $Y$.

Proof. Let $f(A) \subseteq U$ where $U$ is regular open set in $Y$. Since $f$ is continuous, $f^{-1}(U)$ is open set containing $A$. Hence $b c l(A) \subseteq f^{-1}(U)$ (as $A$ is $s g^{*} b$ - closed). Since $f$ is $s g^{*} b$ - closed $f(b c l(A)) \subseteq U$ is $s g^{*} b$ closed set $\Rightarrow \operatorname{bcl}\left(f(b c l(A)) \subseteq U\right.$, Hence $b c l(A) \subseteq U$. So that $f(A)$ is $s g^{*} b$ - closed set in $Y$.

Theorem 3.21. If a map $f:(X, \tau) \rightarrow(Y, \sigma)$ is continuous and closed set and $A$ is $s g^{*} b$ - closed then $f(A)$ is $s g^{*} b$ - closed in $Y$.

Proof. Let $F$ be a closed set of $A$ then $F$ is $s g^{*} b$ - closed set. By theorem $3.20 f(A)$ is $s g^{*} b$ - closed. Hence $f_{A}(F)=f(F)$ is $s g^{*} b$ - closed set of $Y$. Here $f_{A}$ is $s g^{*} b$ - closed and also continuous.

Theorem 3.22. If $f:(X, \tau) \rightarrow(Y, \sigma)$ is closed map and $g:(Y, \sigma) \rightarrow(Z, \eta)$ is $s g^{*} b$ - closed map, then the composition $g \cdot f:(X, \tau) \rightarrow(Z, \eta)$ is $s g^{*} b$ - closed map.

Proof. Let $F$ be any closed set in $(X, \tau)$. Since $f$ is closed map, $f(F)$ is closed set in $(Y, \sigma)$. Since $g$ is $s g^{*} b$ - closed map, $g(f(F))$ is $s g^{*} b$ - closed set in $(Z, \eta)$. That is $g \cdot f(F)=g(f(F))$ is $s g^{*} b$ closed. Hence $g \cdot f$ is $s g^{*} b$ closed map.

Remark 3.23. If $f:(X, \tau) \rightarrow(Y, \sigma)$ is $s g^{*} b$ - closed map and $g:(Y, \sigma) \rightarrow$ $(Z, \eta)$ is closed map, then the composition need not $s g^{*} b$ - closed map .

Theorem 3.24. A map $f:(X, \tau) \rightarrow(Y, \sigma)$ is $s g^{*} b$ - closed if and only if for each subset $S$ of $(Y, \sigma)$ and each open set $U$ containing $f^{-1}(S) \subset U$, there is a $s g^{*} b$ - open set $V$ of $(Y, \sigma)$ such that $S \subset V$ and $f^{-1}(V) \subset U$.

Proof. Suppose $f$ is $s g^{*} b$ - closed. Let $S \subset Y$ and $U$ be an open set of $(X, \tau)$ such that $f^{-1}(S) \subset U$. Now $X-U$ is closed set in $(X, \tau)$. Since $f$ is $s g 6 * b$ - closed, $f(X-U)$ is $s g^{*} b$ - closed set in $(Y, \sigma)$. There fore $V=Y f(X-U)$ is an $s g^{*} b$ - open set in $(Y, \sigma)$. Now $f^{-1}(S) \subset U$ implies $S \subset V$ and $f^{-1}(V)=$ $X-f^{-1}(f(X-U)) \subset X-(X-V)=U$. (ie) $f^{-1}(V) \subset U$.

Conversely, 
Let $F$ be a closed set of $(X, \tau)$. Then $f^{-1}\left(f\left(F^{c}\right)\right) \subset F^{c}$ and $F^{c}$ is an open in $(X, \tau)$. By hypothesis, there exist a $s g^{*} b$ - open set $V$ in $(Y, \sigma)$ such that $f\left(F^{c}\right) \subset V$ and $f^{-1}(V) \subset F^{c} \Rightarrow F \subset f^{-1}(V)^{c}$. Hence $V^{c} \subset f(F) \subset$ $f\left(\left(\left(f^{-1}(V)\right)^{c}\right)^{c} \subset V^{c} \Rightarrow f(V) \subset V^{c}\right.$. Since $V^{c}$ is $s g^{*} b$ - closed, $f(F)$ is $s g^{*} b$ closed. (ie) $f(F)$ is $s g^{*} b$ - closed in $Y$. Therefore $f$ is $s g^{*} b$ - closed.

Theorem 3.25. If $f: X_{1} \times X_{2} \rightarrow Y_{1} \times Y_{2}$ is defined as $f\left(x_{1}, x_{2}\right)=$ $\left(f_{1}\left(x_{1}\right), f_{2}\left(x_{2}\right)\right)$, then $f: X_{1} \times X_{2} \rightarrow Y_{1} \times Y_{2}$ is $s g^{*} b$ closed map.

Proof. Let $U_{1} \times U_{2} \subset X_{1} \times X_{2}$ where $U_{i} \in s g^{*} b c l\left(X_{i}\right)$, for $i=1,2$. Then $f\left(U_{1} \times U_{2}\right)=f_{1}\left(U_{1}\right) \times f_{2}\left(U_{2}\right) \in \operatorname{sg}{ }^{*} b c l\left(X_{1} \times Y_{2}\right)$. Hence $f$ is $s g^{*} b$ - closed set.

Theorem 3.26. Let $h: X \rightarrow X_{1} \times X_{2}$ be $s g^{*} b$ - closed map and Let $f_{i}: X \times X_{i}$ be define as $h(x)=\left(x_{1}, x_{2}\right)$ and $f_{i}(x)=x_{i}$, then $f_{i}: X \times X_{i}$ is $s g^{*} b$ - closed map for $i=1,2$.

Proof. Let $U_{1} \times U_{2} \in X_{1} \times X_{2}$, then $f_{1}\left(U_{1}\right)=h_{1}\left(U_{1} \times X_{2}\right) \in \operatorname{sg}^{*} b c l(X)$, there fore $f_{1}$ is $s g^{*} b$ - closed. Similarly we have $f_{2}$ is $s g^{*} b$ - closed. Thus $f_{i}$ is $s g^{*} b$ - closed map for $i=1,2$.

Theorem 3.27. For any bijection map $f:(X, \tau) \rightarrow(Y, \sigma)$, the following statements are equivalent:

(i) $f^{-1}:(Y, \sigma) \rightarrow(X, \tau)$ is $s g^{*} b$ - continuous.

(ii) $f$ is $s g^{*} b$ - open map.

(iii) $f$ is $s g^{*} b$ - closed map.

Proof. (i) $\Rightarrow$ (ii) Let $U$ be an open set of $(X, \tau)$. By assumption, $\left(f^{-1}\right)^{-1}(U)=$ $f(U)$ is $s g^{*} b$ - open in $(Y, \sigma)$ and so $f$ is $s g^{*} b$ - open.

(ii) $\Rightarrow$ (iii) Let $F$ be a closed set of $(X, \tau)$. Then $F^{c}$ is open set in $(X, \tau)$. By assumption $f\left(F^{c}\right)$ is $s g^{*} b$ - open in $(Y, \sigma)$. Therefore $f\left(F^{c}\right)=f(F)^{c}$ is $s g^{*} b-$ open in $(Y, \sigma)$. That is $f(F)$ is $s g^{*} b$ - closed in $(Y, \sigma)$. Hence $f$ is $s g^{*} b$ - closed.

(iii) $\Rightarrow\left(\right.$ i) Let $F$ be a closed set of $(X, \tau)$. By assumption, $f(F)$ is $s g^{*} b$ closed in $(Y, \sigma)$. But $f(F)=\left(f^{-1}\right)^{-1}(F) \Rightarrow\left(f^{-1}\right)$ is continuous. 


\section{On Semi Generalized $s g^{*} b$-Open Map}

In this section, we introduce semi generalized star $b$ - open map (briefly $s g^{*} b$ open) in topological spaces by using the notions of $s g^{*} b$ - open sets and study some of their properties.

Definition 4.1. Let $X$ and $Y$ be two topological spaces. A map $f$ : $(X, \tau) \rightarrow(Y, \delta)$ is called semi generalized star $b$ - open (briefly, $s g^{*} b$ - open) if the image of every open set in $X$ is $s g^{*} b$ - open in $Y$.

Theorem 4.2. Every open map is $s g^{*} b$ - open but not conversely.

Proof. Let $f:(X, \tau) \rightarrow(Y, \delta)$ is open map and $V$ be an open set in $X$ then $f(V)$ is open in $Y$. Hence $s g^{*} b$ - open in $Y$. Then $f$ is $s g^{*} b$ - open.

The converse of above theorem need not be true as seen from the following example.

Example 4.3. Consider $X=Y=\{a, b, c\}, \tau=\{X, \phi,\{a\},\{b\},\{a, b\}\}$ and $\sigma=\{Y, \phi,\{a, b\}\}$. Let $f:(X, \tau) \rightarrow(Y, \sigma)$ be defined by $f(a)=c, f(b)=$ $a, f(c)=b$. The map is $s g^{*} b$ - open but not open as the image of and $\{a, c\}$ in $X$ is $\{b, c\}$ is not open in $Y$.

Theorem 4.4. A map $f:(X, \tau) \rightarrow(Y, \sigma)$ is $s g^{*} b$ - closed set if and only if for each subset $S$ of $Y$ and for each open set $U$ containing $f^{-1}(S) \subset U$ there is a $s g^{*} b$ - open set $V$ of $Y$ such that $S \subset U$ and $f^{-1}(V) \subset U$.

Proof. Suppose $f$ is $s g^{*} b$ - closed set. Let $S \subset Y$ and $U$ be an open set of $(X, \tau)$ such that $f^{-1}(S) \subset U$. Now $X-U$ is closed set in $(X, \tau)$. Since $f$ is $s g^{*} b$ closed, $f(X-U)$ is $s g^{*} b$ closed set in $(Y, \sigma)$. Then $V=Y-f(X-U)$ is $s g^{*} b$ open set in $(Y, \sigma)$. There fore $f^{-1}(S) \subset U$ implies $S \subset V$ and $f^{-1}(V)=$ $X-f^{-1}(f(X-U)) \subset X-(X-V)=U$. (ie) $f^{-1}(V) \subset U$.

Conversely,

Let $F$ be a closed set of $(X, \tau)$. Then $f^{-1}\left(f\left(F^{c}\right)\right) \subset F^{c}$ and $F^{c}$ is an open in $(X, \tau)$. By hypothesis, there exists a $s g^{*} b$ open set $V$ in $(Y, \sigma)$ such that $f\left(F^{c}\right) \subset V$ and $f^{-1}(V) \subset F^{c} \Rightarrow F \subset\left(f^{-1}(V)^{c}\right.$. Hence $V^{c} \subset f(F) \subset$ $f\left(\left(\left(f^{-1}(V)\right)^{c}\right)^{c}\right) \subset V^{c} \Rightarrow f(V) \subset V^{c}$. Since $V^{c} s g^{*} b$ - closed, $f(F)$ is $s g^{*} b$ closed. (ie) $f(F)$ is $s g^{*} b$ - closed in $(Y, \sigma)$ and there fore $f$ is $s g^{*} b$ - closed.

Theorem 4.5. For any bijection map $f:(X, \tau) \rightarrow(Y, \sigma)$, the following statements are equivalent.

(i) $f^{-1}:(X, \tau) \rightarrow(Y, \sigma)$ is $s g^{*} b$ - continuous. 
(ii) $f$ is $s g^{*} b$ open map.

(iii) $f$ is $s g^{*} b$ - closed map.

Proof. (i) $\Rightarrow$ (ii) Let $U$ be an open set of $(X, \tau)$. By assumption $\left(f^{-1}\right)^{-1}(U)=$ $f(U)$ is $s g^{*} b$ - open in $(Y, \sigma)$. There fore $f$ is $s g^{*} b$ - open map.

(ii) $\Rightarrow$ (iii) Let $F$ be closed set of $(X, \tau)$, Then $F^{c}$ is open set in $(X, \tau)$. By assumption, $f\left(F^{c}\right)$ is $s g^{*} b$ - open in $(Y, \sigma)$. There fore $f(F)$ is $s g^{*} b$ - closed in $(Y, \sigma)$.

Hence $f$ is $s g^{*} b$ - closed.

(iii) $\Rightarrow$ (i) Let $F$ be a closed set of $(X, \tau)$, By assumption $f(F)$ is $s g^{*} b$ closed in $(Y, \sigma)$. But $f(F)=\left(f^{-1}\right)^{-1}(F)$.

Hence $f^{-1}:(X, \tau) \rightarrow(Y, \sigma)$ is $s g^{*} b$-continuous.

\section{References}

[1] Ahmad Al-Omari and Mohd. Salmi Md. Noorani, On Generalized b-closed sets, Bull. Malays. Math. Sci. Soc(2) 32(1) (2009), 19-30

[2] D.Andrijevic, Semi-pre open sets, Mat.Vesnik 38 (1) 1986, 24-32.

[3] D.Andrijevic, b-open sets, Mat.Vesink, 48 (1996), 59-64.

[4] S.P.Arya and T.Nour, Characterizations of s-normal spaces, Indian J.Pure Applied Maths 21 (8) (1990) 717-719.

[5] P.Bhattacharya and B.K.Lahiri, Semi-generalized closed sets on topology, Indian J.Maths 29 (3) (1987) 375-382.

[6] M.Caldas and S.Jafari, On some applications of b-open sets in topological spaces, Kochi J.Math. 24(4) (1998), 681-688.

[7] G.Dimaio and T.Noiri, On s-closed spaces, Indian J-pure appl.math 18 (3) 1987, 226-233

[8] J.Dontchev, On generalized semi- pre open sets, Mem. Fac. Sci. Kochi. Univ. ser. A. math 16 (1995) 35.

[9] Y.Gnanambal, On generalized pre-regular closed sets in topological spaces, Indian J.Pure Appl, Math 28 (1997), 351-360

[10] D.Iyappan \& N.Nagaveni, On semi generalized b-closed set, Nat. Sem. On Mat \& Comp.Sci, Jan (2010), Proc.6

[11] N.Levine, Generalized closed sets in topology, Tend Circ., Mat. Palermo (2) 19 (1970), 89-96.

[12] N.Levine, Semi-open sets and semi-continuity in topological spaces, Amer. Math. Monthly 70 (1963)), 36-41

[13] H.Maki, R.Devi and K.Balachandran, Associated topologies of generalized $\alpha$-closed sets and $\alpha$-generalized closed sets, Mem. Fac. Sci. Kochi. Univ. Ser. A.Math. 15 (1994), 5163.

[14] H.Maki, R.J.Umehara and T.Noiri, Every topological space is pre-T 1/2, Mem. Fac. Sci. Kochi. Univ. Ser. A. Math. 17(1996), 33-42 
[15] A.S.Mashor Abd.El-Monsef.M.E and Ei-Deeb.S.N., On Pre continuous and weak precontinuous mapping, Proc.Math.,Phys.Soc.Egypt, 53 (1982), 47-53.

[16] O.Njastad, On some classes of nearly open sets, Pacific J Math., 15(1965), 961-970.

[17] N.Nagaveni, Studies on generalized on homeomorphisms in topological spaces, Ph.D Thesis, Bharathiar University, Coimbatore 1999

[18] Ravi.O and Ganesan.S, $g$ - closed sets in Topology, International Journal of Computer Science \& Emerging Technologies, Vol 2, Issue3, June 2011, 330-337.

[19] S.Sekar and B.Jothilakshmi, On semi generalized star b-closed set in Topological Spaces, International Journal of Pure and Applied Mathematics, vol. 111, no. 3, (2016).

[20] M.K.R.S. Veerakumar, Between closed sets and g-closed sets, Mem. Fac. Sci. Kochi. Univ. Ser. A: Math., 21 (2000), 1-19. 